\title{
Clustering and Sharing Incentives in BitTorrent Systems
}

\author{
Arnaud Legout \\ I.N.R.I.A. \\ Sophia Antipolis, France \\ arnaud.legout@sophia.inria.fr
}

\author{
Nikitas Liogkas Eddie Kohler Lixia Zhang \\ University of California, Los Angeles \\ Los Angeles, CA, USA \\ \{nikitas,kohler,lixia\}@cs.ucla.edu
}

\begin{abstract}
Peer-to-peer protocols play an increasingly instrumental role in Internet content distribution. Consequently, it is important to gain a full understanding of how these protocols behave in practice and how their parameters impact overall performance. We present the first experimental investigation of the peer selection strategy of the popular BitTorrent protocol in an instrumented private torrent. By observing the decisions of more than 40 nodes, we validate three BitTorrent properties that, though widely believed to hold, have not been demonstrated experimentally. These include the clustering of similar-bandwidth peers, the effectiveness of BitTorrent's sharing incentives, and the peers' high average upload utilization. In addition, our results show that BitTorrent's new choking algorithm in seed state provides uniform service to all peers, and that an underprovisioned initial seed leads to the absence of peer clustering and less effective sharing incentives. Based on our observations, we provide guidelines for seed provisioning by content providers, and discuss a tracker protocol extension that addresses an identified limitation of the protocol.
\end{abstract}

\section{INTRODUCTION}

In just a few years, peer-to-peer (P2P) content distribution has managed to enter the class of applications generating a significant amount of Internet traffic [14]. This widespread adoption of P2P protocols for delivering large data volumes to geographically dispersed peers is arguably due to their scalability and robustness properties. Understanding the mechanisms that affect the performance of such protocols, and designing improved algorithms to overcome existing shortcomings, is critical to the continued success of P2P data delivery. This paper presents a detailed study of BitTorrent, one of the most popular P2P content distribution protocols. We measure BitTorrent's performance in a controlled environment, running real experiments on a private testbed for a variety of scenarios.

There have recently been several attempts to analyze BitTorrent system behavior, as well as experimentally evaluate its fundamental algorithms. Some researchers have formulated analytical models for the problem of efficient data ex-

Technical report

inria-00112066, version 1 - 21 November 2006 change among peers. For example, Yang et al. [23] study the service capacity of BitTorrent-like protocols. They show that it increases exponentially at the beginning of the download session, and scales well with the number of participating peers. In addition, measurement studies of actual download traces have attempted to shed more light into the success of the protocol. For example, Pouwelse et al. [19] study the file popularity, file availability, and content lifetime of numerous download sessions.

However, certain properties of previous studies prevented them from accurately evaluating the dynamics of BitTorrent algorithms and their impact on the overall performance. The analytical models provide valuable insight, but typically make unrealistic assumptions to simplify analysis, such as giving all participants global system knowledge [20,23]; actual download traces can differ substantially from their predictions $[11,19]$. Previous measurement studies have evaluated peers connected to public torrents $[11,12,19]$. These studies provide useful information about the behavior of deployed BitTorrent systems, but the information available from a public torrent is coarse-grained, and does not explain individual peer decisions during the download. A more recent study does evaluate those decisions, but only from the viewpoint of a single peer [15].

In order to overcome these limitations, we evaluate the performance of BitTorrent by running extensive experiments in a controlled environment. In particular, we focus on the so-called choking algorithm for peer selection, which is arguably the driving factor behind the protocol's high performance [8]. This approach allows us to examine the behavior of BitTorrent systems under a microscope, and evaluate the impact of different parameters on system performance. In the process, we validate certain properties of the choking algorithm that are widely believed to hold, but have not been demonstrated experimentally. In addition, we identify new properties and offer insights into the behavior of the choking algorithm in different scenarios, as well as into the impact of proper provisioning of the initial seed on performance.

The contributions of this work are three-fold. First, we demonstrate that the choking algorithm enables good clustering of similar-bandwidth peers, ensures effective sharing incentives by rewarding peers who contribute with high download rates, and achieves high upload utilization for the majority of the download duration. These properties have been hinted at in previous work; this study constitutes their 
first experimental validation. Second, we pinpoint newly observed properties and limitations of the choking algorithm. The new choking algorithm in seed state provides service to all peers uniformly. As a result, if the seed is underprovisioned, clustering is poor and peers tend to finish their downloads at the same time, independently of how much they contribute. Finally, based on our observations, we provide guidelines for seed provisioning by content providers, and discuss a tracker protocol extension that addresses an identified limitation of the protocol, namely the low upload utilization at the beginning of a torrent's lifetime.

The rest of this paper is organized as follows. Section 2 provides a brief description of the BitTorrent protocol and an explanation of the choking algorithm, as implemented in the official BitTorrent client. Section 3 describes our methodology and the rationale behind our experiments, while Section 4 presents our experimental results. Section 5 discusses our proposed seed provisioning guidelines, and the proposed tracker protocol extension. Lastly, Section 6 sets this study in the context of related work, and Section 7 concludes.

\section{BACKGROUND}

BitTorrent is a peer-to-peer content distribution protocol that has been shown to scale with the number of participating peers. In particular, a BitTorrent system capitalizes on the upload capacity of each peer in order to increase the global system capacity as the number of peers increases. A major factor behind BitTorrent's success is its built-in incentive mechanism, as enforced by the choking algorithm, which is intended to motivate peers to contribute data. The rest of this section introduces the terminology used in this paper, describes BitTorrent's operation in detail, and focuses on the choking algorithm in particular.

\subsection{Terminology}

The terminology used in the BitTorrent community is not standardized. For the sake of clarity, we define here the terms used throughout this paper.

- Pieces and Blocks Content transferred using BitTorrent is split into pieces, and each piece is split into multiple blocks. Blocks are the transmission unit in the network, but peers can only share complete pieces with others.

- Interested and Choked We say that peer $A$ is interested in peer $B$, when $B$ has pieces that $A$ does not have. Conversely, peer $A$ is not interested in peer $B$, when $B$ only has a subset of the pieces of $A$. We also say that peer $A$ is being choked by peer $B$, when $B$ has decided not to send any data to $A$. Conversely, peer $A$ is being $u n$ choked by peer $B$, when $B$ is willing to send data to $A$. Note that this does not necessarily mean that peer $B$ is uploading data to peer $A$. It just means that $B$ is willing to upload to $A$, whenever $A$ makes a piece request to $B$.
- Peer Set Each peer maintains a list of other peers, to which it has open TCP connections. We call this list the peer set. This is also known as the neighbor set.

- Local and Remote Peers When we illustrate the choking algorithm below we take the point of view of a single peer that we call local peer. We refer to peers that are in the local peer's peer set as remote peers.

- Leecher and Seed A peer can be in one of two states: the leecher state, when it is still downloading pieces of the content, and the seed state when it has all the pieces and is sharing them with others. In short, we say that a peer is a leecher when it is in the leecher state, and a seed when it is in the seed state.

- Initial Seed The initial seed is the peer that initially offers the content for download. There can be more than one initial seed. In this paper, we consider only the case of a single initial seed.

- Rarest-First Algorithm The rarest-first algorithm is the piece selection strategy used in BitTorrent, also known as the local rarest-first algorithm, since it bases its decisions on limited local knowledge of the torrent. Each peer maintains a list of the number of copies of each piece that peers in its peer set have. It uses this information to define a rarest pieces set, which contains the indices of all the pieces with the least number of copies. This set is updated every time a remote peer in the peer set acquires a new piece, and is consulted for the selection of the next piece to download.

- Choking Algorithm The choking algorithm is the peer selection strategy used in BitTorrent, also known as the tit-for-tat algorithm. We provide a detailed description of this algorithm in section 2.3 .

- Official BitTorrent Client The official BitTorrent client [1], also known as mainline client, was initially developed by Bram Cohen and is now maintained by the company he founded.

\subsection{BitTorrent Operation}

A torrent is a set of peers cooperating to download the same content using the BitTorrent protocol. Prior to distribution, the content is divided into multiple pieces, and each piece into multiple blocks. A metainfo file, also called a torrent file, containing all information necessary for the download process is created. It includes the number of pieces, SHA-1 hashes for all the pieces, and the IP address and port number of the so-called tracker. The hashes are used by peers to verify that a piece has been received correctly. The tracker is the only centralized component of the system. It is not involved in the actual distribution of the content, but rather, it keeps track of all peers currently participating in the download and also collects statistics for all peers. In order to join a torrent, a peer retrieves the metainfo file out of band, usually 
from a well-known website. It then contacts the tracker that responds with a peer set of randomly selected peers, which might include both seeds and leechers. The newly arrived peer starts contacting peers in this set, requesting different pieces.

Most clients nowadays implement the rarest-first algorithm for piece selection. According to that, peers select the next piece to download from their rarest pieces set. They are able to determine which pieces other peers have based on a bitfield message exchanged upon new connections, which contains the list of all pieces a peer has. Peers also send have messages when they successfully receive and verify a new piece. These messages are typically sent to all peers in their peer set.

The selection that determines which peers to exchange data with is made via the so-called choking algorithm. This algorithm gives preference to those peers who upload data at high rates. Once per rechoke period, typically every ten seconds, each peer reconsiders the receiving data rates from all the peers in its peer set. It then selects the fastest ones and uploads only to those for the duration of the period. In BitTorrent parlance, a peer unchokes the fastest uploaders via a regular unchoke, and chokes all the rest. Furthermore, an additional peer is randomly unchoked once every third rechoke period, by means of an optimistic unchoke.

Seeds, who do not need to download any pieces, have to follow a different strategy. Most implementations dictate that seeds unchoke those leechers that download content at the highest rates, in order to better utilize the available seed upload capacity. The official BitTorrent client [1], however, starting with version 4.0.0, has introduced an entirely new algorithm in seed state. In this paper, we perform the first detailed experimental evaluation of this algorithm and show that it contributes to an even more efficient utilization of the seed's bandwidth.

\subsection{Choking Algorithm}

We now describe the choking algorithm in detail, as implemented in the official client, version 4.0.2. This algorithm was introduced to guarantee a high level of data exchange reciprocation, and is one of the main factors behind BitTorrent's sharing incentives: peers that do not contribute should not be able to attain high download rates, since such peers will be choked by others. As a consequence, free-riders, i.e., peers that never upload, should be penalized. The algorithm does not prevent all free-riding $[16,17]$, but we show it performs well in a variety of circumstances.

The choking algorithm is different for leechers and seeds. In leecher state, a fixed number of remote peers are unchoked every rechoke period. This number of parallel uploads is determined by the imposed limit on upload bandwidth, unless specified explicitly by the user. For example, for an upload limit greater than or equal to $15 \mathrm{kB} / \mathrm{s}$ but less than $42 \mathrm{kB} / \mathrm{s}$ this number is four. In the following, we assume that the number of parallel uploads is set to $n$.
In leecher state, the choking algorithm is executed periodically at every rechoke period, i.e., every ten seconds and, in addition, whenever an unchoked and interested peer leaves the peer set, or whenever an unchoked peer switches its interest state. As a consequence, the time interval between two executions of the algorithm can be much shorter than the duration of the rechoke period. Every time the choking algorithm is executed, we say that a new round starts, and the following steps are taken.

1. Interested leechers are ordered according to their observed upload rates to the local peer. However, the local peer ignores leechers that have not sent it any data in the last 30 seconds. These snubbed peers are excluded from consideration in order to guarantee that only contributing peers are unchoked.

2. The $n-1$ fastest of these leechers are unchoked via a so-called regular unchoke.

3. In addition, a candidate peer is chosen at random to be unchoked via a so-called optimistic unchoke.

(a) If the candidate peer is interested in the local peer, it is indeed unchoked via an optimistic unchoke and the round is completed.

(b) Otherwise, the candidate peer is unchoked anyway, but the algorithm repeats step 3 a with a new randomly-chosen candidate.

The round completes when an interested peer is found or when there are no more peers, whichever comes first.

Although more than $n$ peers can be unchoked by the algorithm, only $n$ interested peers can be unchoked in the same round. Unchoking uninterested peers improves reaction time in case one of those peers becomes interested during the following rechoke period: data transfer can begin right away without waiting for the choking algorithm. Optimistic unchokes serve two major purposes. They allow continuous evaluation of the upload contributions of all peers in the peer set, in an effort to discover better partners. They also enable new peers that do not have any pieces yet to bootstrap into the torrent by giving them some first pieces without requiring reciprocation.

For the seed state, older versions of the official client, as well as many current versions of other clients, performed the same steps as in the leecher state with the only difference that the ordering performed in step 1 was based on observed download rates from the seed, rather than upload rates. Consequently, peers with high download capacity were favored independently of their contribution to the torrent, a fact that could be exploited by free-riders [16]. Starting with version 4.0.0, the official client introduced an entirely new choking algorithm in seed state. We are not aware of any other documentation of this new algorithm, nor of any other implementation that uses it. According to this algorithm, the same 
fixed number of $n$ parallel uploads as in the leecher state is performed during every rechoke period. However, the peer selection criteria are now different.

The algorithm is executed periodically at every rechoke period, i.e., every ten seconds, and, in addition, whenever an unchoked and interested peer leaves the peer set, or whenever an unchoked peer switches its interest state. Every time the choking algorithm is executed, a new round starts, and the following steps are taken.

1. The leechers that are interested and unchoked are ordered according to the time they were last unchoked (most recently unchoked peers first). This step only considers leechers that were unchoked recently (less than 20 seconds ago) or that have pending requests for blocks (to ensure that they get the requested data as soon as possible). In case of a tie, leechers are ordered according to their download rates from the seed, fastest ones first. Note that as leechers are not expected to upload anything to seeds, the notion of snubbed peers does not exist in seed state.

2. The number of optimistic unchokes to perform over the duration of the next three rechoke periods, i.e., 30 seconds, is determined using a heuristic. These optimistic unchokes are uniformly spread over this duration, performing $n_{o}$ optimistic unchokes per rechoke period. Due to rounding issues, $n_{o}$ can be different for each of the three rechoke periods. For instance, when the number of parallel uploads is 4 , the heuristic dictates that only 2 optimistic unchokes must be performed in the entire 30 -second period. Thus, 1 optimistic unchoke is performed during each of the first two rechoke periods and none during the last.

3. The first $n-n_{o}$ leechers in the ordered list calculated in step 1 are unchoked via regular unchokes.

Step 1 is the key of the new algorithm in seed state. Leechers are no longer unchoked based on their download rates from the seed, but mainly based on the time of their last unchoke. According to the official client's version notes, this new choking algorithm in seed state aims at reducing the amount of duplicate data a seed needs to upload before it has pushed out a full copy of the content into the torrent.

Some other clients have implemented a super-seeding feature with similar goals, in particular to assist a service provider with limited upload capacity in seeding a large torrent. A seed in super-seeding mode masquerades as a normal leecher with no data. As other peers connect to it, it will advertise a piece that it has never uploaded or that is very rare. After uploading this piece to a leecher, the seed will not advertise any new pieces to that leecher until it sees another peer's advertisement for the piece, indicating that the leecher has indeed shared the piece with others. This algorithm has anecdotally resulted in much higher seeding efficiencies by reducing the amount of redundant pieces uploaded by the seed, and limiting the amount of data sent to peers who do not contribute [2]. A single seed running in this mode is supposed to be able to upload a full copy of the entire content after only uploading $105 \%$ of the content data volume. Since the official client has not implemented this super-seeding feature, our experiments do not measure its effect on the efficiency of the initial seed. Instead, we measure the number of duplicate pieces uploaded by the initial seed when employing the new choking algorithm in seed state.

\section{Methodology}

\subsection{Experimental Setup}

All our experiments were performed with private torrents on the PlanetLab experimental platform [5]. PlanetLab's convenient tools for collecting measurements from geographically dispersed clients greatly facilitated our work. For instance, in order to deploy and launch BitTorrent clients on the PlanetLab nodes, we utilize the pssh tools [4]. PlanetLab nodes are typically not behind NATs, and they keep all their ports open, so each peer in our experiments can be uniquely identified by its IP address. We consciously chose to experiment on private torrents in order to examine both per-peer decisions and the resulting overall torrent behavior. Private torrents allowed us to observe and record the behavior of all peers throughout the torrent's lifetime. It also let us vary experimental parameters, such as upload bandwidth limits of the leechers and the seed. This in turn helped us identify conditions that improve or hinder overall performance and distinguish which factors are responsible for observed behavior.

We performed experiments on different torrent configurations, and repeated each experiment run several times. During each experiment, leechers download a single $113 \mathrm{MB}$ file that consists of 453 pieces, $256 \mathrm{kB}$ each.

PlanetLab's available bandwidth is unusually high for typical torrents; we enforce upload limits on the leechers and seed to model realistic scenarios. However, we do not impose any download limits whatsoever, nor do we attempt to match our upload limits to inherent limitations of PlanetLab nodes. Thus, for example, we might end up imposing a high upload limit on a node that cannot possibly send data that fast, due to network or other problems.

We perform our experiments with a single initial seed, and in all experiments, all leechers join at the same time, simulating a flash crowd scenario. Although the behavior of a torrent might be different with other peer arrival patterns, we are interested in examining peer behavior under circumstances of high load. The initial seed stays connected to the torrent for the duration of each experiment, while leechers disconnect from the torrent immediately after completing their download.

We collect our measurements by utilizing a modified version of the official BitTorrent implementation [1], which we instrumented to record interesting events and peer interactions. The instrumented client is based on version 4.0.2 of the official client, which was released in May 2005. Our client is publicly available for download [3]. The instrumentation 
we collect consists of a log of each message sent or received along with the content of the message, a log of each state change, the rate estimates used by the choking algorithm, and a log of other information, such as internal states of the choking algorithm.

\subsection{Torrent Configurations}

We experimented with several torrent configurations. The parameters we changed from configuration to configuration are the upload limits for the seed and leechers, and the upload bandwidth distribution of leechers. As mentioned before, leecher download bandwidth is never artificially limited, although in some cases, local network characteristics may impose an effective upload or download limit. Since any leecher could potentially download as fast as any other, differences in observed download rates originate solely in BitTorrent's choking algorithm.

We ran experiments with the following configurations:

- Two-class: leechers are divided into two categories with different imposed upload limits. This configuration enables us to observe system behavior in highly bipolar scenarios. Our experiments involve similar numbers of slow peers, with $20 \mathrm{kB} / \mathrm{s}$ upload limit, and fast peers, with $200 \mathrm{kB} / \mathrm{s}$ upload limit.

- Three-class: leechers are divided into three categories with different imposed upload limits. This configuration helps us in identifying the qualitative behavioral differences of more distinct classes of peers. Our experiments involve similar numbers of slow peers, with $20 \mathrm{kB} / \mathrm{s}$ upload limit; medium peers, with $50 \mathrm{kB} / \mathrm{s}$ upload limit; and fast peers, with $200 \mathrm{kB} / \mathrm{s}$ upload limit.

- Uniform: upload limits are imposed on leechers according to a uniform distribution, with a small $5 \mathrm{kB} / \mathrm{s}$ step. In our experiments the slowest leecher has an upload limit of $20 \mathrm{kB} / \mathrm{s}$, the second slowest a limit of $25 \mathrm{kB} / \mathrm{s}$, and so on. This configuration provides insight into the behavior of more homogeneous torrents.

Our graphs in the next section correspond to experiments run with the three-class configuration, but the conclusions we draw accord well with the results of other experiments as well. We stress distinctions where appropriate.

In our experiments, we have considered both a wellprovisioned and an underprovisioned initial seed. Seed upload capacity has already been shown to be critical to performance at the beginning of a torrent's lifetime, before the seed has uploaded a complete copy of the content $[6,15]$. However, it is not clear what the impact of an initial seed with limited capacity is on system properties. Moreover, the capacity threshold below which a limited initial seed adversely impacts the system performance is not trivial to discover. The correct provisioning of the initial seed is fundamental for content providers, in order for them to support torrents that support high system capacity. We attempt to sketch a possible answer in Section 5.1 based on our experimental results.

We also ran preliminary experiments where the initial seed disconnects after uploading an entire copy of the content, but leechers remain connected after they complete their download, becoming seeds for a short period. Peers in these experiments have somewhat lower completion times than configurations with a single seed and immediate leecher disconnection, but appear otherwise similar.

All our experiments are performed with collaborative peers, i.e., peers that never change their upload capacity during a download, or disconnect before receiving a complete copy of the content. However, by considering different upload capacities, and observing the resulting impact on the download rates of peers, we can obtain an initial understanding of BitTorrent system properties in the presence of selfish peers, i.e., peers that want to maximize their utility in the system by abusing protocol mechanisms.

\subsection{Experiment Rationale}

The goal of our experiments is to understand the dynamics and evaluate the efficiency of the choking algorithm. To reach this goal, we consider in this work four metrics.

Clustering: The choking algorithm aims to encourage high peer reciprocation by favoring peers who contribute. Therefore, we expect that peers will more frequently unchoke other peers with similar upload speeds, since those are the peers that can reciprocate with high enough rates. This hypothesis has also been formulated by Qiu et al. [20] in their analytical model of BitTorrent. Consequently, we expect the choking algorithm to converge toward good clustering shortly after the beginning of the download, by grouping together peers with similar upload capacity. This property, however, has never been experimentally verified, and it is not clear whether it is always true. Indeed, let's consider a simple example. Peer $A$ unchokes peer $B$, because $B$ has been uploading data at a high rate to $A$. Yet, in order for peer $B$ to continue uploading to peer $A, A$ should also start sending data to $B$ at a high rate. The only way to initiate such a reciprocative relationship is via an optimistic unchoke. Since optimistic unchokes are performed at random, it is not clear whether $A$ and $B$ will ever get a chance to interact. Therefore, in order to preserve clustering, optimistic unchokes should successfully initiate interactions between peers with similar upload speeds. In addition, such interactions should persist, despite potential disruptions, such as optimistic unchokes by others or network bandwidth fluctuations.

Sharing incentives: A major goal of the choking algorithm is to give peers incentives to share data. The algorithm strives to prevent free-riders from monopolizing the torrent upload capacity, and motivates all peers to contribute, since doing so will improve their own down- 
load rates. Thus, we evaluate the effectiveness of BitTorrent's sharing incentives by measuring how peers' upload contributions affect their download completion time. We expect that the more a peer contributes, the sooner it will complete its download. We do not expect to observe strict data volume fairness, where all peers contribute the same amount of data; peers who upload at high rates may end up contributing much more data than others. However, they should be rewarded by completing their download sooner.

Upload utilization: Upload utilization constitutes a reliable metric of efficiency in peer-to-peer content distribution systems, since the total upload capacity of all peers represents the maximum throughput the system can achieve as a whole. As a result, a peer-to-peer content distribution protocol should aim at maximizing peers' upload utilization. We expect to see this high utilization in BitTorrent systems in our experiments. The question is how far BitTorrent is from optimal upload utilization levels, and which factors can adversely affect utilization in specific scenarios.

Seed service: The new choking algorithm in seed state takes into account the waiting time of peers, in addition to their observed download rates from the seed. Thus, it should be impossible for leechers to monopolize the initial seed, regardless of how fast they can download data. We expect to see an even sharing of the seed upload bandwidth among all peers.

\section{EXPERIMENTAL RESUlts}

We now report the results of representative experiments that demonstrate our main observations. For conciseness, we present only results drawn from the three-class torrent configuration, but our conclusions are consistent with our observations from other configurations.

\subsection{Well-Provisioned Initial Seed}

We first examine a scenario with a well-provisioned initial seed, i.e., a seed that can sustain high upload rates. We expect this to be common for commercial torrents, whose service providers typically make sure there is adequate bandwidth to initially seed the torrent. An example might be Red Hat distributing its latest Linux distribution. Section 4.2 shows that peer behavior in the presence of an underprovisioned initial seed can differ substantially.

We consider an experiment with a single seed and 40 leechers: 13 slow peers ( $20 \mathrm{kB} / \mathrm{s}$ upload limit), 14 medium peers $(50 \mathrm{kB} / \mathrm{s}$ upload limit), and 13 fast peers $(200 \mathrm{kB} / \mathrm{s}$ upload limit). The seed, which is represented as peer 41 in the following figures, is limited to upload $200 \mathrm{kB} / \mathrm{s}$, as fast as a fast peer. These different peer upload limits are imposed in order to model different levels of contribution. The results we report are based on 13 experimental runs. Although the vanilla official BitTorrent implementation would set the

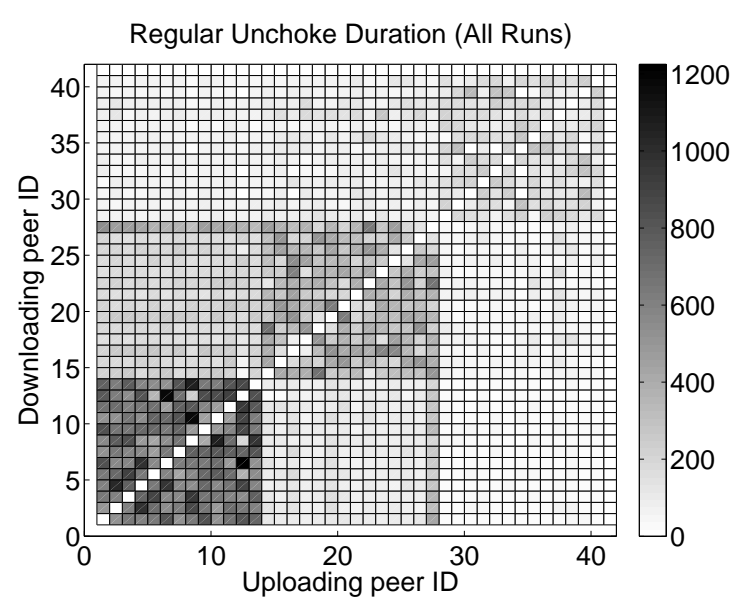

Figure 1: Time duration that peers unchoked each other via a regular unchoke, averaged over all runs. Darker squares represent longer unchoke times. Peers 1 to 13 have a $20 \mathrm{kB} / \mathrm{s}$ upload limit, peers 14 to 27 have a 50 $\mathrm{kB} / \mathrm{s}$ upload limit, and peers 28 to 40 have a $200 \mathrm{kB} / \mathrm{s}$ upload limit. The seed (peer 41) is limited to $200 \mathrm{kB} / \mathrm{s}$. The creation of clusters is clearly visible.

number of parallel uploads based on the imposed upload limit ( 4 for the slow, 5 for the medium, and 10 for the fast peers and the seed), we set this number to 4 for all peers. This ensures homogeneous conditions in the torrent and makes it easier to interpret the results.

\subsubsection{Clustering}

As explained in Section 3.3, we expect to observe clustering based on peers' upload capacities. Figure 1 demonstrates that peers indeed form clusters. The figure plots the total time peers unchoked each other via a regular unchoke, averaged over all runs of the experiment. It is clear that peers in the same class cluster together, in the sense that they prefer to upload to each other. This behavior becomes more apparent when considering a metric such as the clustering index. We define this for a given peer in a given class (fast, medium, or slow) as the ratio of the duration of regular unchokes to the peers of its class over the duration of regular unchokes to all peers. A high clustering index indicates a strong preference to upload to peers in the same class. Figure 2 demonstrates that peers in all classes prefer to unchoke other peers in their own class, thereby forming clusters. Further experiments with upload limits following a uniform distribution also show that peers have a clear preference for peers with similar bandwidths.

Although from Figure 1 it might seem that slow peers show a proportionally stronger preference for their own class, this is an artifact of the experiment. Slow peers take longer to complete their download (as shown in Figure 3), and so perform a higher number of regular unchokes on average than fast peers. Also notice that medium peer 27 interacts frequently with slow peers. This peer's download capacity is inherently limited, as seen in Figure 4 that plots observed peer download speeds over time. As a result, peer 27 stays connected even after all other peers of its class have completed their download. During that last period it has to inter- 


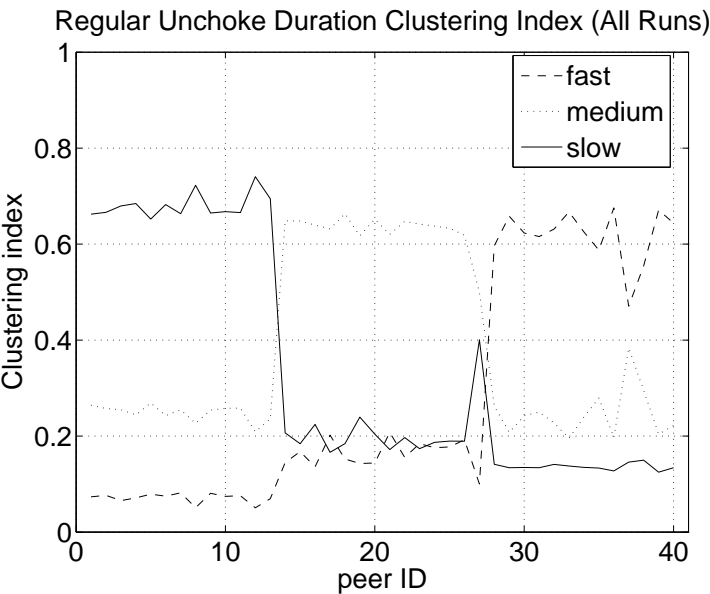

Figure 2: Clustering indices for all peers and all runs, in the presence of a well-provisioned seed. Peers 1 to 13 have a $20 \mathrm{kB} / \mathrm{s}$ upload limit, peers 14 to 27 have a $50 \mathrm{kB} / \mathrm{s}$ upload limit, and peers 28 to 40 have a $200 \mathrm{kB} / \mathrm{s}$ upload limit. The seed (peer 41) is limited to $200 \mathrm{kB} / \mathrm{s}$. Peers show a strong preference to unchoke other peers in the same class.

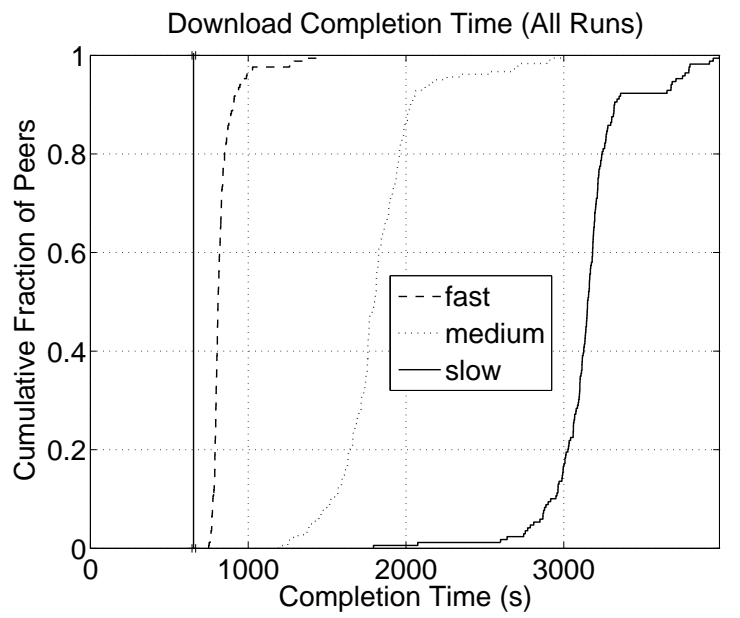

Figure 3: Cumulative distribution of the download completion time for the three different classes of leechers, in the presence of a well-provisioned seed (limited to $200 \mathrm{kB} / \mathrm{s}$ ), for all runs. The vertical line represents the earliest possible time that the download could complete. Fast peers finish much earlier than slow ones.

act with slow leechers, since those are the only ones left. The preference of peer 27 for slow leechers is also evident from the spike anomaly in Figure 2

Figure 1 also shows that reciprocation is not necessarily mutual. Slow peers frequently unchoke medium peers, but the favor is not returned. Indeed, the slow peers unchoked the medium peers for 501,844 seconds, as shown by the centerleft partition that is relatively dark. However, the medium peers unchoked the slow peers for only 273,985 seconds, as shown by the bottom-center partition that is lighter. This lack of reciprocation is due to the fact that slow peers are of little use to medium peers, since they cannot sustain high upload rates.

In summary, the choking algorithm eventually reaches an equilibrium where peers mostly interact with others in the same class, with the occasional exception of optimistic

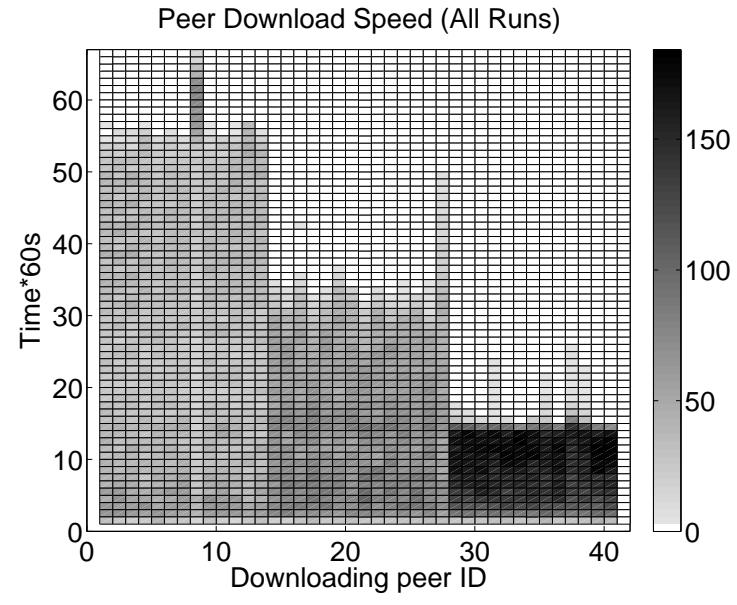

Figure 4: Peer download speeds for all $60-\mathrm{sec}$ time intervals during the download, averaged over all runs. Darker rectangles represent higher speeds. Peers 1 to 13 have a $20 \mathrm{kB} / \mathrm{s}$ upload limit, peers 14 to 27 have a $50 \mathrm{kB} / \mathrm{s}$ upload limit, while peers 28 to 40 have a $200 \mathrm{kB} / \mathrm{s}$ upload limit. The seed (peer 41) is limited to $200 \mathrm{kB} / \mathrm{s}$. Peer 27 achieves lower download rates than the other peers in its class.

unchokes, which are performed randomly. This clustering should help keep the incentives mechanism effective.

\subsubsection{Sharing Incentives}

We now examine whether BitTorrent's choking algorithm provides sharing incentives, in the sense that a peer who contributes more to the torrent is rewarded by completing its download sooner than the rest. Figure 3 demonstrates this to be the case. We plot the cumulative distribution of completion time for the three classes of leechers in the previous experiment. The vertical line in the figure represents the optimal completion time, the earliest possible time that the download could complete. This is the time that the seed has uploaded a complete copy of the content. For this setup, this time is around 650 seconds into the experiment.

Fast leechers complete their download soon after the optimal completion time. Medium and, especially, slow leechers take significantly longer to finish. Thus, contributing to the torrent enables a leecher to enter the fast cluster and receive data at higher rates. This in turn ensures a short download completion time. The choking algorithm does indeed foster reciprocation by rewarding contributing peers. In experiments with upload limits following a uniform distribution, the peer completion time is also uniform; completion time decreases when a peer's upload contribution increases. This further indicates the algorithm's consistent properties with respect to maintaining sharing incentives.

Note, however, that this does not imply any notion of data volume fairness. Fast peers end up uploading significantly more data than the rest. Figure 5, which plots the actual volume of uploaded data averaged over all runs, demonstrates that fast peers are major contributors to the torrent. Most of their bandwidth is expended on other fast peers, per the clustering principle. Interestingly, the slow leechers end up downloading more data from the seed. The seed provides 


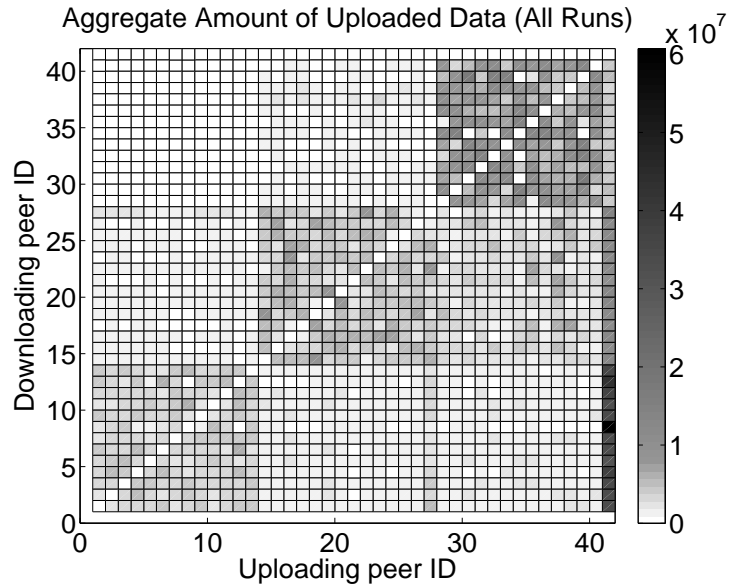

Figure 5: Total number of bytes peers uploaded to each other, averaged over all runs. Darker squares represent more data. Peers 1 to 13 have a $20 \mathrm{kB} / \mathrm{s}$ upload limit, peers 14 to 27 have a $50 \mathrm{kB} / \mathrm{s}$ upload limit, and peers 28 to 40 have a $200 \mathrm{kB} / \mathrm{s}$ upload limit. The seed (peer 41) is limited to $200 \mathrm{kB} / \mathrm{s}$. Fast peers upload much more data than the rest.

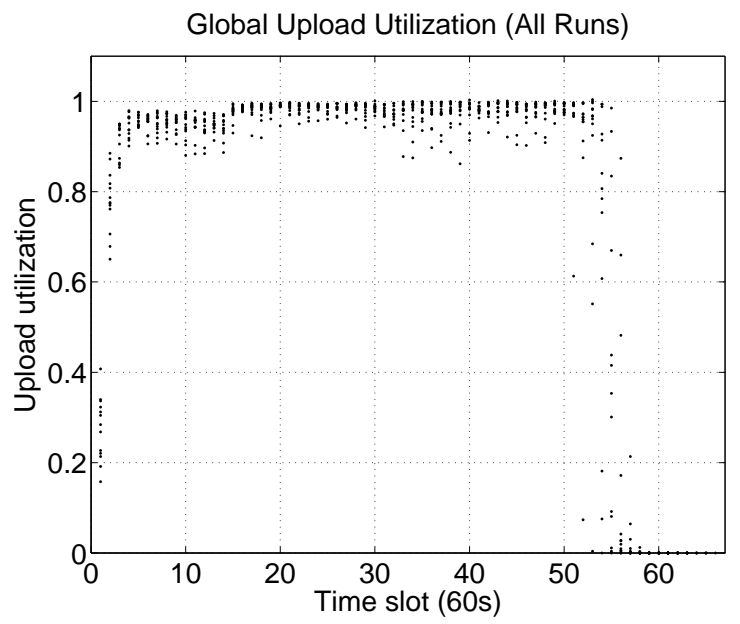

Figure 6: Scatterplot of peers' upload utilization for all 60-sec time intervals during the download, in the presence of a well-provisioned seed (limited to $200 \mathrm{kB} / \mathrm{s}$ ). Each dot represents the average upload utilization over all peers for a given experiment run. Utilization is kept high during most of the download session.

equal service to peers of any class; however, slow peers have more opportunity to download from the seed since they take longer to complete.

In summary, BitTorrent provides effective incentives for peers to contribute, as doing so will reward a leecher with significantly higher download rates. Recent studies $[16,17]$ have shown that limited free-riding is possible in BitTorrent under specific circumstances, although such free-riders do not appear to severely impact the quality of service for other peers. However, these studies do not significantly challenge the effectiveness of sharing incentives enforced by the choking algorithm. Although free-riding is indeed possible, the selfish peers typically achieve lower download rates than they would if they followed the protocol. As a result, if peers wish to obtain as high download rates as possible, it is still in their best interest to conform to protocol guidelines.

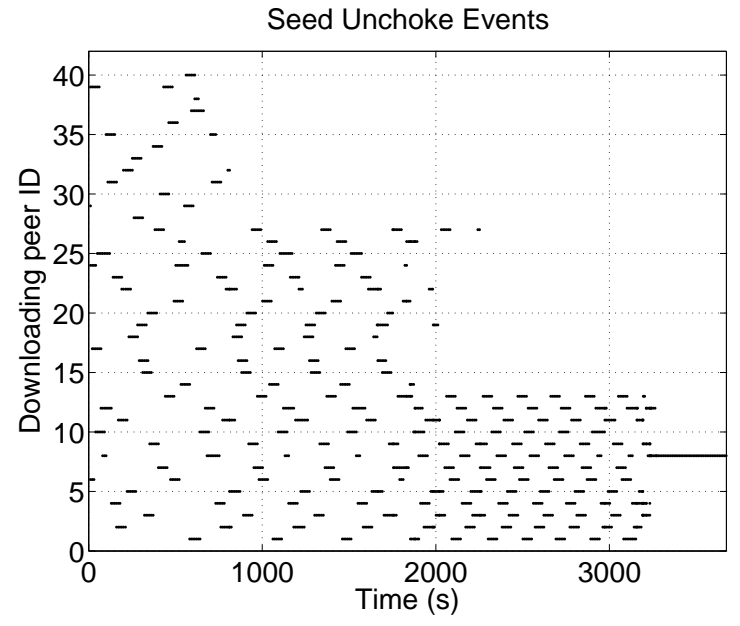

Figure 7: Duration of all unchokes (regular and optimistic) performed by the seed to each peer. Results for a single representative run. Peers 1 to 13 have a $20 \mathrm{kB} / \mathrm{s}$ upload limit, peers 14 to 27 have a $50 \mathrm{kB} / \mathrm{s}$ upload limit, and peers 28 to 40 have a $200 \mathrm{kB} / \mathrm{s}$ upload limit. The seed (peer 41) is limited to $200 \mathrm{kB} / \mathrm{s}$. The seed provides uniform service to all leechers.

\subsubsection{Upload Utilization}

We now turn our attention to performance by measuring whether the choking algorithm can maintain high utilization of the peers' upload capacity. Upload utilization constitutes a reliable metric of efficiency in content distribution systems since the total upload capacity of all peers represents the maximum throughput the system can achieve as a whole. As a result, an efficient protocol should keep peers' upload pipes full at all times.

Figure 6 is a scatterplot of peers' upload utilization in the aforementioned setup. A utilization of 1 represents taking full advantage of the available upload capacity. Utilization for each of the 13 runs is plotted once per minute. The metric is torrent-wide: for each minute, we sum the upload bandwidth used by the peers during that minute, and divide by the upload capacity available over that minute from all peers still connected at the minute's end. The total capacity decreases over time as peers complete their downloads and disconnect. Utilization is low at the beginning and the end of the session, but close to optimal for the majority of the download. It increases slightly after approximately 900 seconds, which corresponds to when fast peers leave the torrent; perhaps the 4-peer limit on parallel uploads restricts fast peers' utilization, or perhaps TCP congestion control's AIMD dynamics have more impact at higher bandwidths. Nevertheless, utilization is good overall.

In summary, the choking algorithm, in cooperation with other BitTorrent mechanisms such as rarest-first piece selection, does a good job of ensuring high utilization of the upload capacity of leechers during most of the download. We discuss a potential solution to low upload utilization at the beginning of a leecher's download in Section 5.2 . 


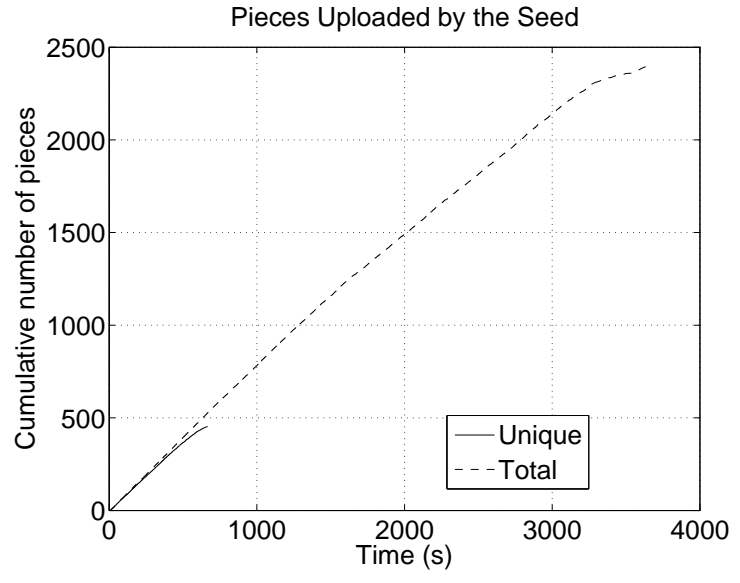

Figure 8: Number of pieces uploaded by the seed, for a single representative run. The Unique line represents the pieces that had not been previously uploaded, while the Total line represents the total number of pieces uploaded so far. The seed is limited to $200 \mathrm{kB} / \mathrm{s}$. We observe a $16 \%$ duplicate piece overhead.

\subsubsection{Seed Service}

The official BitTorrent client's choking algorithm for seeds changed as of version 4.0.0, as described in Section 2.3 The client's version notes claim that this new algorithm "addresses the problem for which super-seeding was created, but without its problems". We performed detailed experiments to study this new algorithm for the first time, and examine this claim.

Figure 7 shows the duration of unchokes, both regular and optimistic, performed by the seed in a representative run of the aforementioned setup. Leechers are unchoked in a uniform manner, regardless of upload speed. Fast peers, those with higher peer IDs, complete their download sooner, after which time the seed divides its upload bandwidth among the remaining leechers. Leecher 8 is the last to complete (as shown in Figure (4), and receives exclusive service from the seed during the end of its download. We see that the new choking algorithm in seed state provides uniform service; this is because it takes each leecher's waiting time into account. As a result, the risk of fast leechers downloading the entire content and quickly disconnecting from the torrent is reduced. Furthermore, this behavior might help mitigate the effectiveness of exploits that attempt to monopolize the seeds [16].

According to anecdotal evidence [2], seeds using the pre4.0.0 choking algorithm might have to upload $150 \%$ to $200 \%$ of the total content size before other peers became seeds. In our experiments, the new choking algorithm avoids this problem. Figure 8 plots the number of pieces uploaded by the seed during the download session for a representative run. 527 pieces are sent out before an entire copy of the content (453 pieces) has been uploaded. Thus, the duplicate piece overhead is around $16 \%$, indicating that the new choking algorithm in seed state avoids unnecessarily uploading duplicate pieces to a certain extent. This number was consistent across all our experiments. However, to the best of our

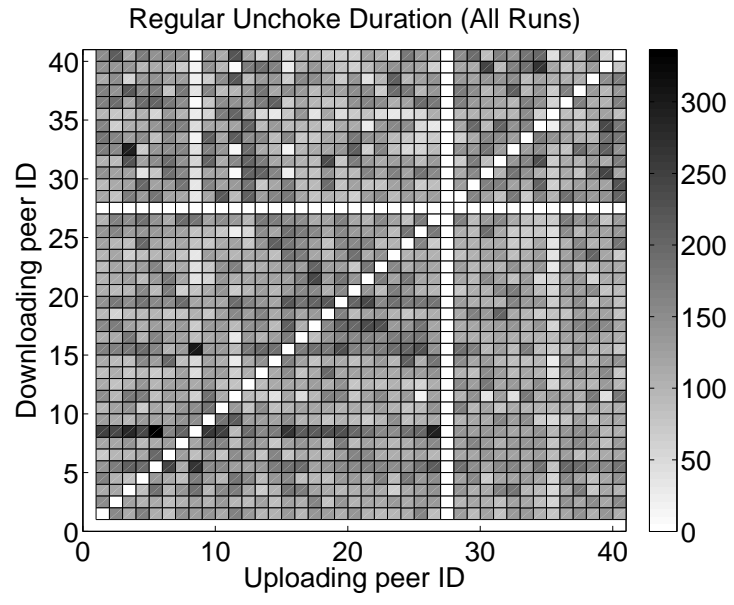

Figure 9: Time duration that peers unchoked each other via a regular unchoke, averaged over all runs. Darker squares represent longer unchoke times. Peers 1 to 12 have a $20 \mathrm{kB} / \mathrm{s}$ upload limit, peers 13 to 26 have a $50 \mathrm{kB} / \mathrm{s}$ upload limit, and peers 28 to 40 have a $200 \mathrm{kB} / \mathrm{s}$ upload limit. The seed (peer 27) is limited to $100 \mathrm{kB} / \mathrm{s}$. There is no discernible clustering.

knowledge, there has been no experimental evaluation of the corresponding overhead in the old choking algorithm in seed state, so it is not clear how much of an improvement this is; we will investigate this in future work.

Nevertheless, $16 \%$ duplication represents an opportunity for improvement. The existing implementation always issues requests for pieces in the rarest pieces set in the same order, if the set contains more than one. As a result, leechers might end up requesting the same rarest piece from the seed at approximately the same time. It would arguably be preferable for leechers to request rarest pieces in random order, so that the probability of multiple leechers requesting the same piece at the same time is minimized.

In summary, the new choking algorithm in seed state uniformly distributes seed upload capacity among leechers, independently of their upload contributions. Our results also show that it incurs a reasonably low duplicate piece overhead.

\subsection{Underprovisioned Initial Seed}

We now turn our attention to a scenario with an underprovisioned initial seed and demonstrate that the seed upload capacity is critical to performance during the beginning of a torrent's lifetime. The experiment we present here involves a single seed and 39 leechers, 12 slow, 14 medium, and 12 fast. The initial seed in this case, represented as peer 27 in the following figures, is limited to $100 \mathrm{kB} / \mathrm{s}$, not $200 \mathrm{kB} / \mathrm{s}$. We set the number of parallel uploads again to 4 for the seed and all the leechers. The results we present are based on $8 \mathrm{ex}-$ periment runs, and are consistent with our observations for experiments with other torrent configurations. We show that peer behavior in the presence of an underprovisioned initial seed is substantially different than with a well-provisioned initial seed. 


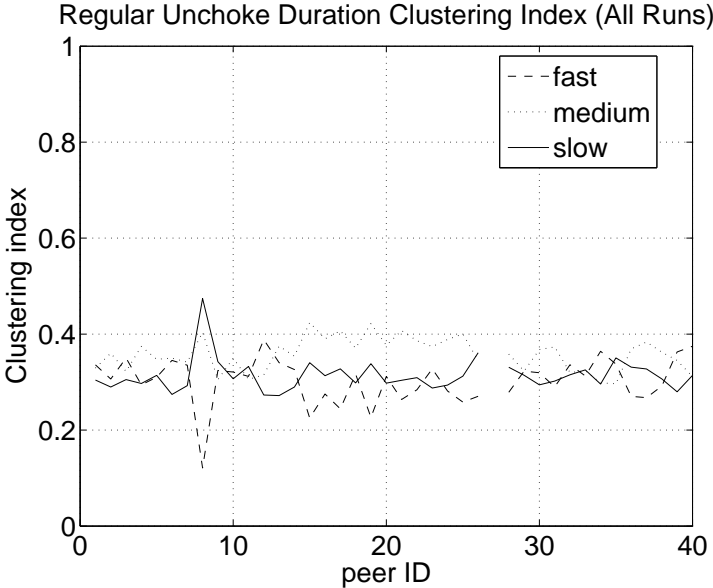

Figure 10: Clustering indices for all peers in the presence of an underprovisioned seed. Peers 1 to 12 have a $20 \mathrm{kB} / \mathrm{s}$ upload limit, peers 13 to 26 have a $50 \mathrm{kB} / \mathrm{s}$ upload limit, and peers 28 to 40 have a $200 \mathrm{kB} / \mathrm{s}$ upload limit. The seed (peer 27) is limited to $100 \mathrm{kB} / \mathrm{s}$. Peers do not show a clear preference to unchoke other peers in any particular class.

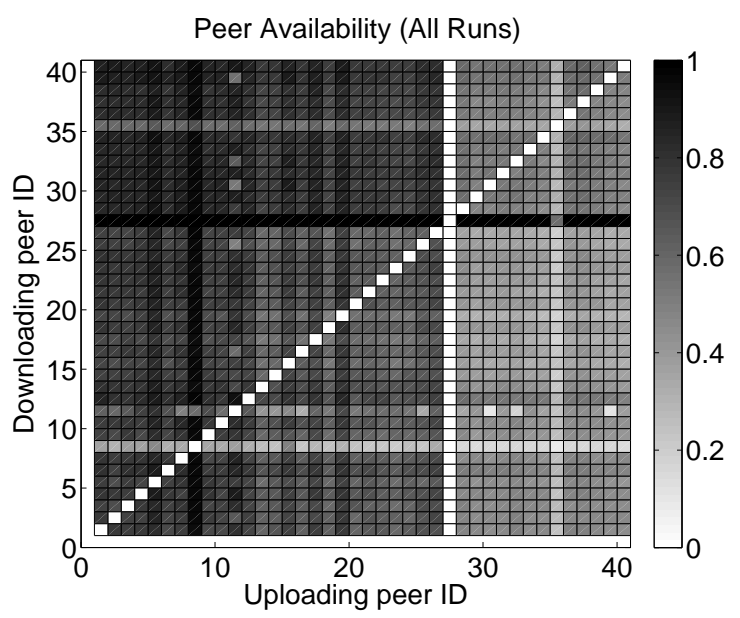

Figure 11: Normalized interested time duration for each peer pair, averaged over all runs. Darker squares represent higher peer availability. Peers 1 to 12 have a $20 \mathrm{kB} / \mathrm{s}$ upload limit, peers 13 to 26 have a $50 \mathrm{kB} / \mathrm{s}$ upload limit, and peers 28 to 40 have a $200 \mathrm{kB} / \mathrm{s}$ upload limit. The seed (peer 27) is limited to $100 \mathrm{kB} / \mathrm{s}$. Fast peers have poor peer availability to all other peers.

\subsubsection{Clustering}

Figure 9 shows the total time peers unchoked each other via a regular unchoke, averaged over all runs of the experiment. In contrast to Figure 1 there is no discernible clustering among peers in the same class. The lack of clustering in the presence of an underprovisioned initial seed becomes more apparent when considering the clustering index metric mentioned in Section 4.1.1 Figure 10 shows the clustering indices of all peers. They are all very similar, indicating a lack of preference to unchoke peers in any particular class. Compare this to Figure 2, where the preference for peers in the same class is evident.

Figure 11 explains this behavior by plotting the peer availability of each peer to each other peer, averaged over all runs of the experiment. We define the peer availability of a down- loading peer $Y$ to an uploading peer $X$ as the ratio of the time $X$ was interested in $Y$ to the time that $Y$ spent in the peer set of $X$. A peer availability of 1 means that the uploading peer was always interested in the downloading peer, while a peer availability of 0 means that the uploading peer was never interested in the downloading peer.

From the figure we can see that the fast peers have poor peer availability to all other peers. The seed is uploading new pieces at a low rate, so even if the seed uploaded only to fast peers, those fast peers would quickly replicate every piece as it was completed, remaining idle for the rest of the time. Thus, fast peers are not interested in others most of the time. The same is not true for slow peers, since they upload even more slowly than the seed. In addition, when a fast leecher is unchoked by a slow leecher, it will always reciprocate with high rates, and thereby be preferred by the slow leecher. As a result, fast peers will get new pieces even from medium and slow peers. Thus, fast peers prevent clustering by taking up slower peers' unchoke slots and thus breaking any clusters that might be starting to form. Further experiments with other torrent configurations, including one with the initial seed further limited to $20 \mathrm{kB} / \mathrm{s}$, confirm this conclusion.

In summary, when the initial seed is underprovisioned, the choking algorithm does not enable peer clustering. We study in the next section how this lack of clustering affects the effectiveness of sharing incentives.

\subsubsection{Sharing Incentives}

Given the lack of clustering, we now examine whether BitTorrent's choking algorithm still provides incentives to share even in the presence of an underprovisioned initial seed. In particular, we examine whether fast peers still complete their download sooner than others. Figure 12 shows that this is no longer the case. Most peers complete their download at approximately the same time. Most points in the tail of the figure are due to a single slow peer, peer 8 , which in every run completed its download last. This PlanetLab node has a poor effective download speed independently of the choking algorithm, likely due to network problems or machine overload. All other peers achieve completion times below 2000 seconds in every experiment. Clearly, seed upload capacity is the performance bottleneck. Once the seed finishes uploading a full copy of the content, all peers complete soon thereafter. Since uploading data to other peers does not shorten a peer's completion time, BitTorrent's sharing incentives are ineffective here.

Fast peers are again the major contributors in the torrent, but in this case their upload bandwidth is expended equally across other fast peers and slower peers alike. Figure 13 plots the amount of uploaded data between each peer pair. A quick visual inspection shows that the fast peers contribute roughly equally to all other peers, and that fast peers made most contributions, while the slow ones made the least.

In summary, when the initial seed is underprovisioned, the choking algorithm does not provide effective incentives to 


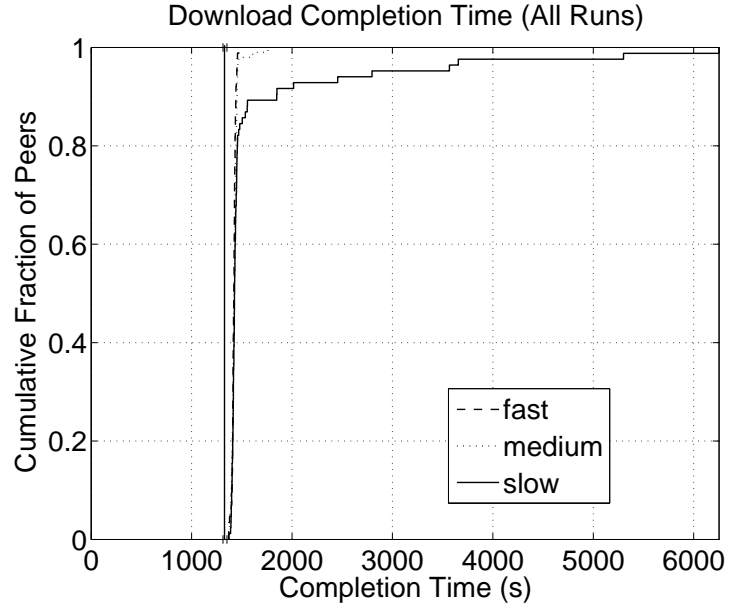

Figure 12: Cumulative distribution of the download completion time for the three different classes of leechers, in the presence of an underprovisioned seed (limited to $100 \mathrm{kB} / \mathrm{s}$ ), for all runs. The vertical line represents the earliest possible time that the download could complete. Most peers complete at approximately the same time, soon after the seed finishes uploading a full copy of the content.

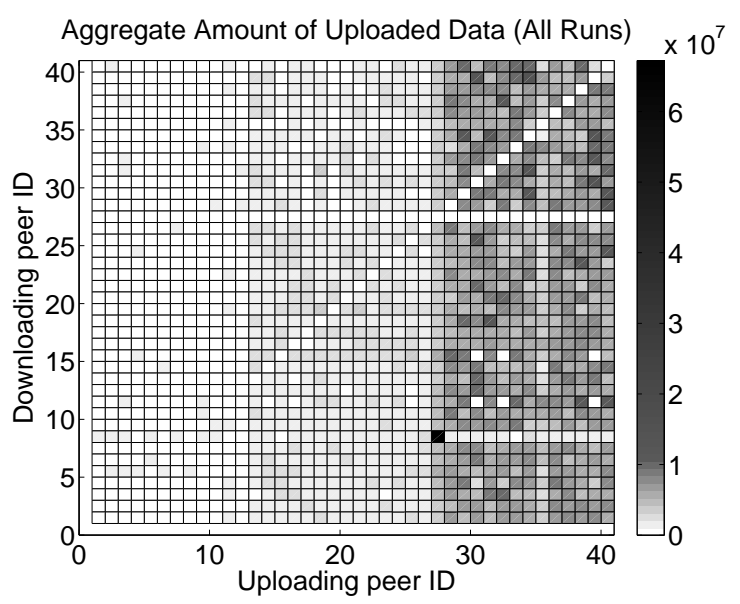

Figure 13: Total number of bytes peers uploaded to each other, averaged over all runs. Darker squares represent more data. Peers 1 to 12 have a 20 $\mathrm{kB} / \mathrm{s}$ upload limit, peers 13 to 26 have a $50 \mathrm{kB} / \mathrm{s}$ upload limit, and peers 28 to 40 have a $200 \mathrm{kB} / \mathrm{s}$ upload limit. The seed (peer 27) is limited to 100 $\mathrm{kB} / \mathrm{s}$. Fast peers upload much more data than the rest, distributing those data evenly among all peers.

share. However, the available upload capacity of fast peers is effectively utilized to efficiently replicate the pieces being sent by the initial seed.

\subsubsection{Upload Utilization}

We now evaluate the impact of an underprovisioned initial seed on overall BitTorrent system performance. Figure 14 plots peers' upload utilization. Even with a slow seed, upload utilization remains relatively high. Leechers manage to exchange data productively among themselves once new pieces are downloaded from the slow seed, so that the lack of clustering does not degrade torrent performance significantly. Interestingly, the BitTorrent design seems to lead the system to do the right thing: fast peers contribute their bandwidth to re-

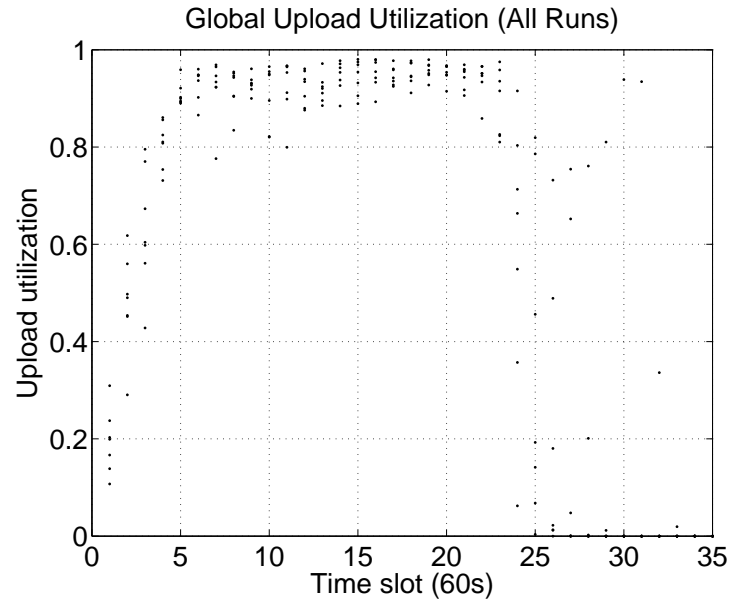

Figure 14: Scatterplot of peers' upload utilization for all 60-sec time intervals during the download, in the presence of an underprovisioned seed (limited to $100 \mathrm{kB} / \mathrm{s}$ ). Each dot represents the average upload utilization over all peers for a given experiment run. Utilization is kept at acceptable levels despite the seed limitation.

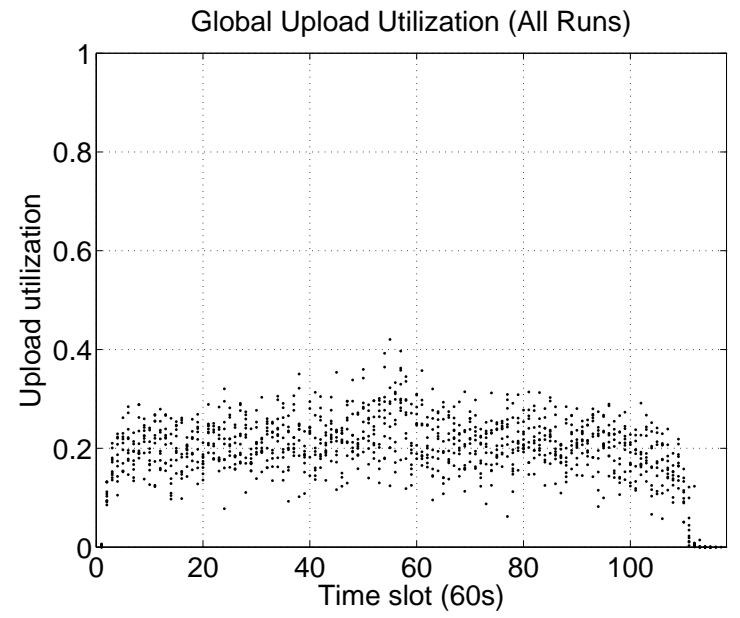

Figure 15: Scatterplot of peers' upload utilization for all 60-sec time intervals during the download, in the presence of a severely underprovisioned seed (limited to $20 \mathrm{kB} / \mathrm{s}$ ). Each dot represents the average upload utilization over all peers for a given experiment run. Utilization is poor when the seed is very slow.

duce the burden on the initial seed, helping disseminate the available pieces to slower peers. Indeed, this destroys clustering, but it improves the torrent efficiency, which is a reasonable decision given the situation.

We also experimented with a seed limited to an upload capacity of $20 \mathrm{kB} / \mathrm{s}$. With this extremely low seed upload speed, there are few new pieces available to exchange at any point in time, and each new piece gets disseminated rapidly after it is retrieved from the seed. Fig. 15]shows that the overall upload utilization is now low; slow peers exhibit slightly higher utilization than the rest, since they do not need many available pieces to use up their available upload capacity.

In summary, even in situations where the initial seed is underprovisioned, the global upload utilization can be high. However, our experiments only involve collaborative users, 
who do not try to adapt their upload speed according to a utility function of the observed download speed. On the other hand, in a selfish environment with an underprovisioned seed, one might expect a lower upload utilization due to the lack of sharing incentives.

\section{Discussion}

We discuss two limitations of the choking algorithm that we identified in the section 4 , seed upload capacity is fundamental to the proper operation of the incentives mechanism, and at the beginning of the download session peers take some time to reach full upload utilization.

\subsection{Seed Provisioning}

When the initial seed is underprovisioned, the choking algorithm does not lead to clustering of similar-bandwidth peers. Even without clustering, however, we observed high upload utilization. Interestingly, in the presence of a slow initial seed, the protocol makes fast leechers contribute to the download of all other peers, fast or slow, as evidenced in Figure 13, thereby improving the overall torrent capacity.

However, whenever feasible, one should engineer adequate initial seed capacity in order to allow fast leechers to achieve high performance. Our results show that the lack of clustering occurs when fast peers cannot maintain their interest in other fast peers. In order to avoid this situation, the initial seed should at least be able to upload data at a speed that matches that of the fastest peers in the torrent. This suggestion is simply a rule-of-thumb guideline, and assumes that the service provider knows a priori the maximum upload capacity of the peers that may join the torrent in the future. In practice, reasonable bounds could be derived from measurements or from an analysis of deployed network technologies. Further research is needed to evaluate the exact impact of seed capacity. We are currently developing an analytical model that attempts to express the effect of initial seed capacity on the overall torrent performance.

\subsection{Tracker Protocol Extension}

When a new leecher first joins the torrent, it connects to a random subset of already-connected peers that are returned by the tracker. However, in order to reach its optimal bandwidth utilization, this new peer needs to exchange data with those peers that have a similar upload capacity to itself. If there are few such peers in the torrent, it may take some time to discover them, since this process has to be done via random optimistic unchokes that take place only once every 30 seconds.

Consequently, it might be preferable in such a scenario to employ the tracker to assist in matching similar-bandwidth leechers. In this manner, the discovery period duration could decrease and the upload utilization would be high even at the beginning of a peer's download. The new peer could report its upload capacity to the tracker when joining the torrent. This speed can be the one configured in the client software, or possibly the actual maximum upload speed measured during previous downloads. The tracker would then reply with a random subset of peers as usual, along with their upload capacity. The new leecher would have the option of performing optimistic unchokes first to peers with upload capacity similar to its own, in an effort to discover the best partners sooner.

With this new tracker protocol extension, if the peer set contains only a few leechers with similar upload capacity, they will be discovered quickly. However, since the tracker still returns a random subset of peers independently of the advertised upload capacity, there is no benefit for a peer to lie. If it does so, other peers who connect to it will discover this fact quickly, and choke the lying leecher, since it would not be able to sustain appropriate upload rates. In a collaborative environment, however, the tracker might even want to return peers based on their advertised upload capacity, as also proposed in [6], in order to speed up cluster creation even more. Although this extension is promising, further research is required to verify that it will work as expected.

\section{RELATED WORK}

There has been a fair amount of work on the performance and behavior of BitTorrent systems. Bram Cohen, the protocol's creator, has described BitTorrent's main mechanisms and their design rationales [8]. Several analytical studies have formulated models for BitTorrent-like protocols. Biersack et al. [7] propose an analysis of three content distribution models: a linear chain, a tree, and a forest of trees. They discuss the impact of the number of pieces and the number of parallel uploads for each model, and claim that the optimal efficiency is achieved using 3 to 5 parallel uploads. Yang et al. [23] study the service capacity of BitTorrent systems and show that it increases exponentially at the beginning of the torrent, and scales well with the number of peers. Qiu et al. [20] extend this work by providing an analytical solution to a fluid model of BitTorrent. Their results show BitTorrent's high upload utilization. However, their model assumes peer selection based on global knowledge of all peers in the torrent, as well as uniform distribution of pieces. Moreover, they do not consider the dynamics of the choking algorithm. Massoulie et al. [18] introduce a probabilistic model and claim that system performance does not depend critically on the rarest-first piece selection strategy. Lastly, Fan et al. [9] characterize the complete design space of BitTorrent-like protocols by providing a mathematical model that captures the trade-off between high performance and fairness. As previously mentioned, whereas all these models provide valuable insight into the behavior of BitTorrent systems, unrealistic assumptions limit their applicability in real scenarios.

Other researchers have relied on simulations to understand BitTorrent's properties. Felber et al. [10] compare different peer and piece selection strategies in different torrent configurations. Bharambe et al. [6] utilize a discrete event simulator to evaluate upload utilization and bit-level fairness. They 
find that the protocol scales very well and that the rarest-first algorithm outperforms alternative piece selection strategies. However, they do not evaluate a peer set larger than 15 peers, whereas the official implementation has a default value of 80. This limitation may have an important impact on the behavior of the protocol, as the accuracy of the piece selection strategy is affected by the peer set size. Moreover, they do not consider the new version of the choking algorithm in seed state. Tian et al. [22] propose a simple analytical model to study BitTorrent's performance and validate it using simulations. They also propose and evaluate a new peer selection strategy during the last phase of a download session, in order to enable more peers to complete their download after the departure of all the seeds.

There have been several measurement studies that examined actual BitTorrent traffic. Izal et al. [12] identify several peer characteristics in the tracker log for the Redhat Linux 9 ISO image, including the percentage of peers completing the download, load on the seeds, and geographical spread of participating peers. They observe a correlation between uploaded and downloaded amount of data. Pouwelse et al. [19] study the file popularity, file availability, download performance, and content lifetime on a formerly popular tracker website. They observe that, although BitTorrent can efficiently handle large flash crowds, the central tracker component could potentially be a bottleneck. A more recent study by Guo et al. [11] demonstrates that peer performance fluctuates widely in small torrents, and that high-bandwidth peers tend to contribute less to the torrents. Inter-torrent collaboration is proposed as an alternative to providing extra incentives for leechers to stay connected after the completion of their download. Lastly, Legout et al. [15] run extensive experiments on real torrents, from the viewpoint of a single peer. They show that the rarest-first and choking algorithms play a critical role in BitTorrent's performance. In particular, they show that the rarest-first piece selection strategy approximates an optimal piece selection strategy after a complete copy of the content has been uploaded, and that the choking algorithm fosters reciprocation. They claim that the replacement of the current choking algorithm by a bit-level tit-for-tat algorithm is not appropriate, as proposed by other researchers [13]. However, they do not identify the reasons behind the properties of the choking algorithm, and fail to examine its dynamics due to the single peer viewpoint.

Furthermore, researchers have looked into the feasibility of circumventing BitTorrent mechanisms to free-ride on the torrent. Shneidman et al. [21] were the first to demonstrate that BitTorrent exploits are indeed feasible. Jun et al. [13] argue that the choking algorithm cannot prevent free-riding, and propose a new algorithm as a replacement. Liogkas et al. [16] design and implement three exploits that allow a peer that does not contribute to maintain high download rates under specific circumstances. However, they show that, even though such peers can sometimes obtain more bandwidth, there is no considerable degradation of the overall system's quality of service. Lastly, Locher et al. [17] extend this work by demonstrating that limited free-riding is feasible even in the absence of seeds. They also describe how free-riding is possible in BitTorrent sharing communities.

Our work differs from all previous studies in its approach and results. We perform the first extensive experimental study of BitTorrent in a controlled environment, by monitoring all the peers in the torrent, and examining the behavior of the BitTorrent system in a variety of scenarios. Our results validate protocol properties that have not been demonstrated experimentally previously, as well as new properties with respect to the impact of the initial seed on performance.

\section{Conclusion}

In this paper we presented the first experimental investigation of BitTorrent systems that links per-peer decisions and overall torrent behavior. Our results validated three BitTorrent properties that, though widely believed to hold, have not been demonstrated experimentally. We showed that the choking algorithm enables clustering of similar-bandwidth peers, ensures effective sharing incentives by rewarding peers who contribute with high download rates, and achieves high upload utilization for the majority of the download duration. We also examined the properties of the new choking algorithm in seed state and the impact of initial seed capacity on the overall BitTorrent system performance. In particular, we showed that an underprovisioned initial seed does not enable clustering of peers and does not guarantee effective sharing incentives. However, we showed that even in such a case, the choking algorithm guarantees an efficient utilization of the available resources by enforcing fast peers to help other peers with their download. Based on our observations, we offered guidelines for content providers regarding seed provisioning, and discussed a tracker protocol extension that addresses an identified limitation of the protocol.

This work opens up many avenues for future work. We are currently developing an analytical model to express the effect of the seed capacity on torrent performance. It would also be interesting to run experiments with the old choking algorithm in seed state and compare its properties to the new algorithm. In addition, we would like to investigate the impact of different number of regular and optimistic unchokes on the protocol's performance and fairness properties. It has recently been argued [9] that the trade-off between these two kind of unchokes is critical. The current values used by the protocol are intuition-based engineering choices; we would like to conduct a systematic evaluation of system behavior under different values for these parameters.

\section{REFERENCES}

[1] BitTorrent, Inc. http://www.bittorrent.com

[2] BitTorrent Specification wiki. http://wiki.theory.org/BitTorrentSpecification/

[3] Instrumented BitTorrent client. http://www-sop.inria.fr/planete/Arnaud.Legout/Proje 
[4] Parallel versions of openssh tools. http://www.theether.org/pssh/

[5] PlanetLab open platform. http: //www.planet-lab.org.

[6] A. R. Bharambe, C. Herley, and V. N. Padmanabhan. Analyzing and Improving a BitTorrent Network's Performance Mechanisms. In Proc. of Infocom'06, Barcelona, Spain, April 2006.

[7] E. W. Biersack, P. Rodriguez, and P. Felber. Performance Analysis of Peer-to-Peer Networks for File Distribution. In Proc. of the International Workshop on Quality of future Internet Services (QofIS'04), Barcelona, Spain, September 29October 1, 2004.

[8] B. Cohen. Incentives Build Robustness in BitTorrent. In Proc. of the Workshop on Economics of Peer-to-Peer Systems (P2PEcon'03), Berkeley, CA, June 2003.

[9] B. Fan, D.-M. Chiu, and J. C. Lui. The Delicate Tradeoffs in BitTorrent-like File Sharing Protocol Design. In Proc. of ICNP'06, Santa Barbara, CA, November 2006.

[10] P. A. Felber and E. W. Biersack. Self-scaling Networks for Content Distribution. In Proc. of the International Workshop on Self-* Properties in Complex Information Systems (Self*'04), Bertinoro, Italy, May 31-June 2, 2004.

[11] L. Guo, S. Chen, Z. Xiao, E. Tan, X. Ding, and X. Zhang. Measurements, Analysis, and Modeling of BitTorrent-like Systems. In Proc. of IMC'05, Berkeley, CA, October 2005.

[12] M. Izal, G. Urvoy-Keller, E. W. Biersack, P. Felber, A. A. Hamra, and L. Garcés-Erice. Dissecting BitTorrent: Five Months in a Torrent's Lifetime. In Proc. of PAM'04, Antibes Juan-les-Pins, France, April 2004.
[13] S. Jun and M. Ahamad. Incentives in BitTorrent Induce Free Riding. In Proc. of the Workshop on Economics of Peer-toPeer Systems (P2PEcon'05), Philadelphia, PA, August 2005.

[14] T. Karagiannis, A. Broido, N. Brownlee, kc claffy, and M. Faloutsos. Is P2P dying or just hiding? In Proc. of Globecom'04, Dallas, TX, November 29-December 3, 2004.

[15] A. Legout, G. Urvoy-Keller, and P. Michiardi. Rarest First and Choke Algorithms Are Enough. In Proc. of IMC'06, Rio de Janeiro, Brazil, October 2006.

[16] N. Liogkas, R. Nelson, E. Kohler, and L. Zhang. Exploiting Bittorrent For Fun (But Not Profit). In Proc. of IPTPS'06, Santa Barbara, CA, February 2006.

[17] T. Locher, P. Moor, S. Schmid, and R. Wattenhofer. Free Riding in BitTorrent is Cheap. In Proc. of HotNets-V, Irvine, CA, November 2006. To appear.

[18] L. Massoulie and M. Vojnovic. Coupon Replication Systems. In Proc. of SIGMETRICS'05, Banff, Canada, June 2005.

[19] J. Pouwelse, P. Garbacki, D. Epema, and H. Sips. The BitTorrent P2P file-sharing system: Measurements and Analysis. In Proc. of IPTPS'05, Ithaca, NY, February 2005.

[20] D. Qiu and R. Srikant. Modeling and Performance Analysis of BitTorrent-Like Peer-to-Peer Networks. In Proc. of SIGCOMM'04, Portland, Oregon, August 30-September 3, 2004.

[21] J. Shneidman, D. Parkes, and L. Massoulie. Faithfulness in Internet Algorithms. In Proc. of the Workshop on Practice and Theory of Incentives and Game Theory in Networked Systems (PINS'04), Portland, OR, September 2004.

[22] Y. Tian, D. Wu, and K. W. Ng. Modeling, Analysis and Improvement for BitTorrent-Like File Sharing Networks. In Proc. of Infocom'06, Barcelona, Spain, April 2006.

[23] X. Yang and G. de Veciana. Service Capacity of Peer to Peer Networks. In Proc. of Infocom'04, Hong Kong, China, March 2004. 